\section{Brain, Behavior and Evolution}

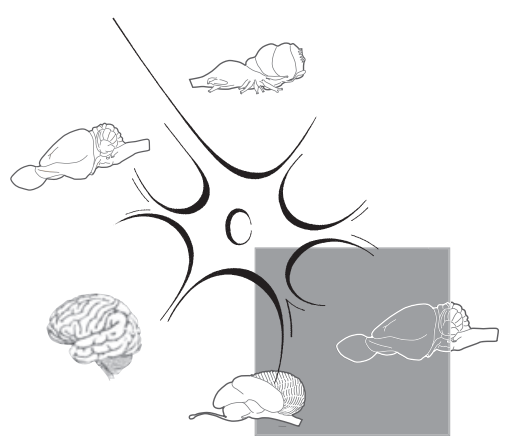

\section{Keywords}

Model · Diversity · Brain · Behavior . Evolution

With renewed recognition of the value of addressing neuroscience questions in a range of species [e.g., Hale, 2014; Striedter, 2019; Bolker, 2019] and the exciting and rapidly developing genetic tools for doing so [Heidenreich and Zhang, 2016; Mardis, 2017], it is an important time to consider how to support the use of diverse species in research. Neuroscience at present is Balkanized by species, with scientists generally working with just one taxon. This is true not only for those working in traditional genetic model organisms, but also for many others using less common models. Better leveraging of species diversity may be best accomplished not by forming additional scientific communities focused on one species, but rather by increasing the capacity of scientists to adopt new study systems in their laboratories and to move between animal models more readily. Such flexibility would promote investigators taking their questions of interest beyond the constraints of a particular study organism. More broadly, greater familiarity with the work performed in systems other than one's primary animal model would be

\title{
Toward Diversification of Species Models in Neuroscience
}

\author{
Melina E. Hale ${ }^{a, b}$ \\ ${ }^{a}$ Department of Organismal Biology and Anatomy, The University of Chicago, \\ Chicago, IL, USA; ${ }^{\mathrm{b}}$ Marine Biological Laboratory in Woods Hole, Woods Hole, MA, USA
}

healthy for the field, promoting idea generation and appreciation of efforts in other models.

\section{Development and Integration of Alternative Species Models}

Below I expand upon two critical efforts to support greater use of diverse species in neuroscience and thus allow investigators more flexibility to try experiments in a range of systems. I will firstly consider tool development and expanding capabilities for work in diverse species. Although there are only a few traditional genetic model organisms, they differ markedly in size and other characteristics. Their variation has required a diversity of approaches and technologies that are often highly specialized for mouse, fly, zebrafish, or another species or even for a particular usecase for work in one species. The development of new tools that would accommodate a wider range of species and preparations is exciting to consider. The second consideration is expanding species diversity in neuroscience research through education and training. For neuroscientists to be more adaptable in the organisms that they use in research, development of training and other resources to broaden exposure to diverse species and how to work with them should be considered. This is needed not only for scientists who are extending their work into new models, but also for scientists who are familiar with diverse species but have less experience with emerging tools and techniques. Ultimately tools, techniques, and training should come together, allowing scientists to better leverage research opportunities in diverse species with state-of-the-art approaches.

\section{Tool Development and Expanding}

Capabilities for Work in Diverse Species

Technological advances in microscopy and other neuroscience tools are often made with a particular model organism or use in mind. This approach tends to optimize an instrument or technique for a specific purpose, but the tool's use on other species, for other purposes, is often limited. Variation among organisms is immense, not only in the size and shape of the nervous systems and neurons themselves, but also in the properties of the tissues that surround the nervous system and, for live preparations, the physiological demands of those tissues. For both technique and tool development, considering design objectives that include enabling breadth of use and engaging scientists using diverse models to understand needs should be en-

\section{KARGER}

(c) 2019 S. Karger AG, Basel
Melina E. Hale

Department of Organismal Biology and Anatomy (ANAT 401)

The University of Chicago, 900 E. 57th Street

Chicago, IL 60637 (USA)

E-Mail mhale@uchiago.edu 
couraged. Expanded and easier access to emergent technology would greatly enhance scientists' ability to work in diverse organisms and broaden their range of preparations.

The BRAIN Initiative's focus on tool development has provided significant opportunities to advance research in diverse species through improvements in instrumentation, software, and other capabilities. BRAIN Initiative leaders have highlighted needs to identify and access brain cell types, diagram circuits, assess whole-brain activity and link it to behavior, determine foundational principles of brain function, and develop approaches to better understand and treat the human brain [Jorgenson et al., 2015]. Jorgenson and colleagues were explicit about the value of work in non-human organisms, both vertebrates and invertebrates, and taking advantage of the "unique strengths of diverse species and experimental systems."

One example of implementing these ideas is the National Science Foundation's major BRAIN Initiative program: Developing a National Research Infrastructure for Neuroscience (NeuroNex). The NeuroNex solicitation in 2016 called for the development of neurotechnology hubs and theory teams to address BRAIN Initiative aims and highlighted work in diverse animals. NSF awarded nine NeuroNex Neurotechnology Hub awards, two NeuroNex Theory Teams awards and, in addition, six NeuroNex Innovation awards, which also aim to contribute to neurotechnology resources. Based on summaries available through the National Science Foundation (e.g., https://www.nsf.gov/news/news_ summ.jsp?cntn_id=242652), many of these projects explicitly aim to develop capabilities that can be used in a wide range of species to examine neuron activity and circuit structure or to make genetic tools available in non-model organisms. One example is the NeuroNex Hub at Cornell University where multiphoton imaging with a wider field of view and deeper penetration will allow imaging in small but intact organisms [Wang et al., 2018] and in vivo imaging in a broad range of species. Another example is the Miniscope Neuronex Center, where head-mounted brain imaging technology is allowing functional imaging during relatively unrestrained behaviors. Through the work of this Center techniques currently being developed for rats and mice may soon become applicable to larger species, such as marmosets [Scott et al, 2018].
As technological resources continue to be developed, the application of emerging technology in new preparations and with diverse questions and organisms is important for optimizing the use of BRAIN Initiative resources. Exploring a new resource can be time consuming and expensive, especially away from one's home institution, and when there is some chance that the technology will not work as hoped. Strategic planning for dissemination of new neurotechnology, with a particular focus on scientists and laboratories that may not have the funding levels to acquire new technology or travel to use it elsewhere, is key. Supporting the use of emerging technology at convenient, open-access facilities would be one way to support and encourage the adoption of new approaches. Expertise available at such facilities can be incredibly valuable in helping new users develop and troubleshoot preparations and approaches.

Ultimately, an online database that relates the properties of organisms, brains, and neurons to preparation approaches and appropriate technologies would be incredibly helpful in simplifying the first stages of determining how to study a particular species. For example, it would be useful to find the region of experimentation space where a given technology has been used for research on organisms/nervous systems:

- of a particular size

- from a particular environment (marine, freshwater, terrestrial, etc.)

- with particular organismal properties (hard bodied, soft bodied, transparent, opaque, etc.)

- in particular tissues (brain, spinal cord, periphery, sense organs, muscle, etc.)

- using in vivo/in vitro preparations or fixed tissue

Such a resource could provide a mechanism for finding research opportunities and may also identify gaps where emergent technology should be useful for particular research but has not yet been applied. Finding those gaps would help to determine where to incentivize adoption or new development of technology.

\section{Expanding Species Diversity in Neuroscience Research through Education and Training}

A key question for diversifying the species used in neuroscience research is: How can the community gather and share information on diverse species and the oppor- tunities they provide for research? As students enter neuroscience from a wide range of backgrounds and with limited exposure to taxonomy and biodiversity, it is likely that most budding neuroscientists come to the field with a narrow lens on species models. In addition, as pointed out by Ballen and Greene [2017], even for college students who have had biodiversity training, the focus of that training is usually on taxonomic ranks and nomenclature, rather than biological diversity. Recent efforts to approach diversity teaching within a phylogenetic context [e.g. Hillis et al., 2014; Ballen and Greene, 2017] provide a higher-level framework within which to think about taxonomic groups and their relationships. Also available are web-based resources that focus on classic morphological characteristics in diverse taxa (e.g., encyclopedia of life, eol.org; tree of life, tol. org) and that characterize taxonomic diversity through short, unique DNA sequence barcodes (e.g., Barcode of Life Database, BOLD, http://www.boldsystems. org; International Barcode of Life, iBOL, ibol.org). In-person courses on biodiversity and online options for exploring diversity could likewise be of value to the community.

Biodiversity resources that serve neuroscientists, either in person or online, would be particularly useful if they included a focus on function and behavior, as well as practical information for working in the live organisms. In particular, watching animals move in laboratory demonstrations or, more practically, on video can provide critical insights that are much harder to grasp through other forms of documentation. Even more valuable is watching animals in natural or naturalistic environments as they interact with their physical surroundings and other organisms. Information about the ease of animal acquisition and care, generation time, optical accessibility, and other characteristics crucial to experimental feasibility of work can further aid investigators in deciding whether a particular question or area of study is worth pursuing, given the constraints on a researcher or lab (space, time, costs, and other factors).

Already, some experience-based courses and workshops provide valuable training. For example, the long-running zebrafish short course at The Marine Biological Laboratory in Woods Hole (MBL) has trained generations of researchers in this model organism. In addition, a range 
of other courses at the MBL bring the latest technology and diverse applications together [Nishi et al., 2016]. Short courses or workshops combining the exploration of diverse species and approaches to studying them could be considered. Outside of a formalized educational experience, opportunities to work with potential model organisms and related equipment at institutions with open facilities and visitor programs could create a pipeline for identifying potential models and the techniques needed to work with them productively.

\section{Future Considerations}

As model systems diversify, an additional critical question should be considered: How can study species and related resources be maintained over time as interest in their use waxes and wanes? This is an important question even for many models that are already in use. We currently risk losing expertise and techniques developed outside of the major genetic mod-

\section{References}

Ballen CJ, Greene HW. Walking and talking the tree of life: why and how to teach about biodiversity. PLoS Biol. 2017 Mar;15(3):e2001630.

Bolker JA. Selection of models: evolution and the choice of species for translational research. Brain Behav Evol. 2019;93(2-3):82-91.

Hale ME. Mapping circuits beyond the models: integrating connectomics and comparative neuroscience. Neuron. 2014 Sep;83(6):12568.

Heidenreich M, Zhang F. Applications of CRISPR-Cas systems in neuroscience. Nat Rev Neurosci. 2016 Jan;17(1):36-44. el organisms, as such knowledge tends to exist only in a small number of labs. Not considering the impact of such loss, or ways to prevent it and protect past and current investment, would be shortsighted. The future potential of a system can be difficult to predict, as discovery and technological innovation may open up avenues for research that were previously unrecognized. As a community we need better approaches to retaining the resources and skills needed to work in an inactive system, such that future researchers can readily revitalize this research direction. There are many possible ways to address this question but, hopefully, options will be thoughtfully considered so that we do not drift inattentively towards an unclear and unplanned future.

The US BRAIN Initiative is a great example of the neuroscience community thinking about future needs and aspirations for the field, and articulating solutions. The time is right for performing a similar exercise, though smaller in scale, for planning how to utilize and support the use of diverse model species (traditional and new) in neuroscience research.

\section{Acknowledgements}

Thanks to the symposium organizers, Georg Striedter and Todd Preuss, and to all of the participants from whom I've learned so much. In particular, I thank G. Striedter for his help with editing. Thanks to Prof. Mark Westneat for his expertise on phylogenetics.

\section{Disclosure Statement}

The author has no financial interests to declare. The author is a former Co-Interim Director and is currently a Fellow at the Marine Biological Laboratory in Woods Hole.

\section{Funding Sources}

This commentary was informed by work on the NSF award IOS1638400, Research Coordination Network: ENSEMBLE - Enabling Neuroscience in Species Models That Broadly Leverage Evolution (M.E. Hale, PI).
Hillis DM, Sadava DE, Hill RW, Price MV. Principles of life. London: Macmillan Higher Education; 2014.

Jorgenson LA, Newsome WT, Anderson DJ, Bargmann CI, Brown EN, Deisseroth K, et al. The BRAIN Initiative: developing technology to catalyse neuroscience discovery. Phil Trans R Soc Lond B Biol Sci. 2015;370(1668): 20140164.

Mardis ER. DNA sequencing technologies: 20062016. Nat Protoc. 2017 Feb;12(2):213-8.

Nishi R, Castañeda E, Davis GW, Fenton AA, Hofmann HA, King J, et al. The global challenge in neuroscience education and training: the MBL perspective. Neuron. 2016 Nov; 92(3):632-6.
Scott BB, Thiberge SY, Guo C, Tervo DG, Brody CD, Karpova AY, et al. Imaging Cortical Dynamics in GCaMP Transgenic Rats with a Head-Mounted Widefield Macroscope. Neuron. 2018 Dec;100(5):1045-1058.e5.

Striedter GF. Variation across species and levels: implications for model species research. Brain Behav Evol. 2019;93(2-3):57-69.

Wang T, Ouzounov DG, Wu C, Horton NG, Zhang B, Wu CH, et al. Three-photon imaging of mouse brain structure and function through the intact skull. Nat Methods. 2018 Oct;15(10):789-92. 